\title{
Last Observed Value Imputation Technique
}

National Cancer Institute

\section{Source}

National Cancer Institute. Last Observed Value Imputation Technique. NCI Thesaurus.

Code C132341.

A data imputation technique that populates analysis values with the subject's last

recorded nonmissing observation. 